\title{
Effects of non-supervised exercise interventions on bone mineral density in adult women: a systematic review and meta-analysis
}

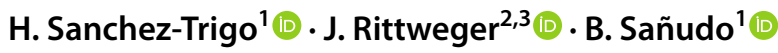

Received: 24 May 2021 / Accepted: 16 February 2022 / Published online: 26 February 2022

(c) International Osteoporosis Foundation and National Osteoporosis Foundation 2022

\begin{abstract}
Mini abstract Osteoporosis is a major health issue worldwide. This study analyzes the effects of non-supervised osteoporosis prevention programs on bone mineral density. Non-supervised exercise increases femoral neck and lumbar spine bone mineral density in adult women. Thus, it might be effective for preventing or treating osteoporosis or osteopenia in this population. Introduction Osteoporosis is a major health issue worldwide. Social distancing measures due to COVID-19 have hindered the chances to take part in supervised osteoporosis prevention exercise programs. The purpose of the present study is to systematically review and meta-analyze the effects of non-supervised osteoporosis prevention exercise programs on bone mineral density (BMD) in adult women.

Methods A comprehensive search of electronic databases $(n=7)$ was conducted including (a) prospective randomized controlled trials (RCTs) comparing at least one exercise group vs. a control group with sedentary lifestyle or sham exercises; (b) baseline and follow-up BMD values, or BMD changes from baseline, at any skeletal site; (c) women over 30 years old; and (d) non-supervised exercise programs only. Subgroup analyses were performed for menopausal status, intervention duration, type of exercise, and osteopenia/osteoporosis status.

Results Ten studies were included $(n=668)$. Random effect analyses showed that unsupervised exercise had beneficial effects on lumbar spine (LS) BMD with standardized mean difference (SMD) $=0.40$ (95\% confidence interval (CI): 0.03-0.77), and femoral neck (FN) BMD with SMD $=0.51$ (95\% CI: 0.16-0.85). Unsupervised exercise increased LS (SMD $=0.73$ (95\% CI: 0.13-1.33)) and FN BMD (SMD = 0.85 (95\% CI: 0.33-1.37)) in women with osteopenia/osteoporosis, but not in healthy counterparts.

Conclusion Non-supervised exercise improves FN and LS BMD in adult women. Beneficial effects of exercise on FN and LS BMD might be more pronounced in those with poor bone health compared with healthy counterparts. More RCTs prescribing non-supervised, osteogenic exercise are required in this population. It is necessary to investigate the efficacy of remote/ assistive technologies for delivering and monitoring non-supervised exercise interventions.
\end{abstract}

Keywords Exercise, Non-supervised, COVID-19, Osteoporosis, Bone

\section{Introduction}

H. Sanchez-Trigo

fstrigo@us.es

1 Departamento de Educación Física y Deporte, Universidad de Sevilla, 41013 Seville, Spain

2 Institute of Aerospace Medicine, German Aerospace Center (DLR), 51147 Cologne, Germany

3 Department of Pediatrics and Adolescent Medicine, University of Cologne, 50931 Cologne, Germany
Osteoporosis is a disease characterized by low bone mass and microarchitectural deterioration of bone tissue, leading to increased bone fragility and fracture risk [1]. It is estimated that 25.5 million people in the European Union and 10.2 million people in the USA are affected by osteoporosis [2]. One in three women above 50 years old will suffer an osteoporotic fracture [3]. Fractures represent a large burden on health care systems. In Europe, costs associated with hospitalization and aftercare due to osteoporotic fractures amounted to 34 billion euro in 2010 [4]. These costs are expected to increase twofold by 2050 based on the expected 
population aging [5]. It is therefore of importance to develop interventions to prevent and treat osteoporosis that could reduce costs and mitigate the social impact of this highly prevalent disease. Such interventions should involve little risks and be sustainable over long periods.

Exercise is considered a safe, low-cost, non-pharmaceutical intervention for the prevention and treatment of osteoporosis [6, 7]. It has been shown that modalities such as resistance training or impact exercise prevent or reverse about $1 \%$ of bone loss per year at the lumbar spine (LS) and femoral neck (FN) in both pre- and postmenopausal women [8]. Bones adapt to mechanical loads with an osteogenic response, if these loads exceed those of habitual activities [9]. However, the optimum exercise program to improve bone health remains to be determined [10]. Exercises that involve weight-bearing loads and impacts, such as hopping and jumping, have shown improvements in bone mineral density (BMD) and microarchitecture [11]. As for the magnitude of these improvements, a previous meta-analysis reported a 0.37 standardized mean difference (SMD) at the LS (equivalent to mean changes in the BMD of $+0.006 \mathrm{~g} /$ $\mathrm{cm}^{2}$ in the intervention group and $-0.010 \mathrm{~g} / \mathrm{cm}^{2}$ in the control group), and 0.33 SMD at the FN (equivalent to mean changes of $+0.004 \mathrm{~g} / \mathrm{cm}^{2}$ in the intervention group and $-0.012 \mathrm{~g} / \mathrm{cm}^{2}$ in the control group), with interventions lasting $11.7 \pm 5.7$ months [12].

While this evidence in favor of exercising for improving bone health is of importance, the question of adherence to such exercise-based osteoporosis prevention programs arises. A meta-analysis found a dropout rate of $21 \%$ and a compliance of $76 \%$ in supervised exercise interventions targeting bone mineral density in adults [13]. A recent review identified lack of time and transportation as the most common barriers to attending supervised exercise programs for osteoporosis prevention [14]. An obvious strategy to overcoming these barriers is the development of non-supervised home-based exercise programs to eliminate transportation needs, reduce costs, and allow for flexible scheduling [15].

In March 2020, when the World Health Organization declared COVID-19 a global pandemic, new challenges to engage in supervised exercise programs arose. COVID-19 is an infectious disease caused by a coronavirus discovered in 2019 known as SARS-CoV-2 [16]. Social distancing measures are negatively affecting people's physical activity behaviors and increasing sedentary time, deteriorating therefore physical health and well-being [17]. Inevitably, "lockdown" strategies have hindered the chances to participate in supervised osteoporosis prevention exercise programs [18], and it could well be that elements of social distancing persevere after the end of the COVID-19 pandemic.

Therefore, if the aim is to utilize exercise as a non-pharmacological strategy to improve bone health and prevent osteoporosis in adult women, and if participation is limited for the abovementioned reasons, then one should look for alternative means of providing exercise interventions. Nonsupervised interventions lend themselves as an ideal solution, because they can be carried out at home with complete time flexibility. Indeed, a recent study analyzed the consequences of COVID-19 lockdown on the adherence to exercise programs specifically designed for women with postmenopausal osteoporosis, and found that participation increased in non-supervised home training [19]. The question arises, however, whether such home-based exercise programs are effective enough to warrant formal, evidencebased recommendation. To our knowledge, no previous systematic review and meta-analysis has exclusively focused on the effects of non-supervised osteoporosis prevention exercise programs on BMD in pre- and postmenopausal women. The purpose of the present study is to systematically review and meta-analyze the effects of non-supervised exercise programs on BMD in this population.

\section{Methods}

\section{Search strategy}

The reporting of this systematic review was guided by the standards of the Preferred Reporting Items for Systematic Review and Meta-Analysis (PRISMA) Statement [20] and was registered in advance in the International Prospective Register of Systematic Reviews (PROSPERO) with ID CRD42020189320.

The following bibliographic databases were searched: PubMed, PsycInfo, Web of Science, Scopus, SportDiscus, Cochrane, and Cinahl. Databases were systematically searched for articles published up to August 16, 2021, without language restrictions. The search strategy comprised of a combination of population (i.e., women), intervention (i.e., physical activity, exercise, training, walking), and outcome (i.e., BMD, bone, osteoporosis). Accordingly, the search term was ("physical activity" OR exercise OR training OR walking) AND (bone OR osteoporosis OR BMD) AND women. All citations were entered into a reference management software (Mendeley Desktop Software, version 1.19.4, Elsevier). Duplicates were excluded automatically, and the remaining studies were screened by title and abstracts according to the eligibility criteria.

\section{Inclusion and exclusion criteria}

Studies were included if they met the following criteria: (a) prospective randomized controlled trials (RCTs) comparing at least one exercise group vs. a control group with sedentary lifestyle or sham exercises; (b) baseline and follow-up BMD values, or BMD changes from baseline assessed by 
dual-energy X-ray absorptiometry (DXA) or dual photon absorptiometry (DPA), at any skeletal site; (c) women over 30 years old; (d) non-supervised exercise programs only.

The following studies we excluded: (a) follow-up studies, review articles, case reports, editorials, conference abstracts, and letters; (b) mixed-gender cohorts without separate BMD analyses for each sex; (c) studies that included women with diseases that relevantly affect bone metabolism (e.g., hyperthyroidism, type I diabetes mellitus, renal or hepatic disease) [21], or undergoing chemo- and/or radiotherapy; (e) studies combining exercise with pharmacological therapy.

\section{Data extraction}

Titles and abstracts were screened by one reviewer (H. S.-T.) to exclude irrelevant studies. Two reviewers (H. S.-T. and B. S.) independently evaluated full-text articles and extracted data from the included studies. Disagreement was resolved by discussion between these two reviewers. Extracted data included subjects' characteristics, sample size for each group, length of intervention, compliance, exercise modality, BMD values with standard deviations (SD) at different skeletal sites (i.e., FN and LS) at baseline and study completion, attrition, and adverse event data. Corresponding authors were contacted for any missing data.

\section{Outcome measures}

While initially outcomes of interest were BMD at any skeletal site commonly assessed to diagnose osteoporosis, only LS and FN were included in the meta-analyses due to data availability in the selected studies. Besides, LS and FN are common osteoporotic fracture sites [22].

\section{Exercise interventions}

Non-supervised exercise interventions for treating or preventing osteoporosis were classified according to the following six categories [23]:

- Static weight-bearing (SWB), e.g., single-leg standing.

- Dynamic weight-bearing exercise low force (DWBLF), e.g., walking, and Tai chi.

- Dynamic weight-bearing exercise high force (DWBHF), e.g., jogging, jumping, running, dancing and vibration platform.

- Non-weight-bearing exercise low force (NWBLF), e.g., low load, high repetition strength training.

- Non-weight-bearing exercise high force (NWBHF), e.g., progressive resisted strength training.

- Combination (COMB); more than one of the above exercise interventions.

\section{Quality assessment}

All studies included were independently assessed for risk of bias by two independent raters (H. S.-T. and B. S.) using the Physiotherapy Evidence Database (PEDro) scale [24]. The methodological quality of the selected studies was classified as follows: $\geq 7$, high; $5-6$, moderate; $<5$, low [25].

\section{Statistical analysis}

All statistical analyses were performed using the software Review Manager (RevMan, Version 5.4, The Cochrane Collaboration, 2020). Effect size (ES) values were considered the standardized mean differences (SMDs), computed as

$\mathrm{SMD}=\frac{\operatorname{mean}\left(D_{\mathrm{Int}}\right)-\operatorname{mean}\left(D_{\mathrm{Ctr}}\right)}{\mathrm{SD}\left(D_{\mathrm{Int}, \mathrm{Ctr}}\right)}$

where $D_{\mathrm{Int}}$ and $D_{\mathrm{Ctr}}$ are the post-pre differences in the intervention and control groups, respectively, and $D_{\mathrm{Int}, \mathrm{Ctr}}$ is the post-pre difference of the combined groups [26]. If the SDs of the post-pre differences in the intervention and control groups were not reported in the selected studies, then it was calculated from confidence intervals (CIs), standard error, or $P$-value of the absolute change of BMD using standardized formulae [27]. If none of these data were available, the following formula was employed:

$\mathrm{SD}=\sqrt{\mathrm{SD}_{\text {pre }}^{2}+\mathrm{SD}_{\text {post }}^{2}-\left(2 \cdot r \cdot \mathrm{SD}_{\text {pre }} \cdot \mathrm{SD}_{\text {post }}\right)}$

where $r$ is the correlation coefficient that describes how similar the pre- and post-measurements were across participants [27]. Estimated values for this correlation coefficient for the intervention and control groups at LS and FN have been reported elsewhere [12]. Sensitivity analyses were performed by replacing correlation coefficients with 0.7 to explore whether weaker coefficients had any effect on overall findings.

The magnitude of the SMD in the social sciences can be interpreted as small, $\mathrm{SMD}=0.2$; medium, $\mathrm{SMD}=0.5$; and large, $\mathrm{SMD}=0.8$ [28]. CI for the SMDs were computed at the $95 \%$ confidence level. The variability in interventions employed in the different studies requires the use of a random effects model [26]. Heterogeneity between studies was examined using the $Q$ statistic. Consistency of intervention effects across studies was assessed using the $I^{2}$ statistic. Generally, an $I^{2}$ below $40 \%$ is considered low heterogeneity, 30 to $60 \%$ represents moderate, and 50 to $90 \%$ substantial heterogeneity [27]. Z-score was computed to test for overall effect. The rank correlation between effect estimates and their standard errors was conducted using the $t$-test and Kendall's $\tau$ statistic [29]. The $P$-value $<0.05$ was defined 
as the significant level for all tests. Subgroup analyses were performed for menopausal status, intervention duration, type of exercise, and osteopenia/osteoporosis status. Sensitivity analyses were conducted omitting studies of low methodological quality to determine the robustness of the overall results. It is important to note that one of the included studies [30] reports results for premenopausal subjects and postmenopausal subjects separately; therefore, it was also included separately in the meta-analyses, and labeled as "SugiyamaPOSM" for postmenopausal data and "SugiyamaPREM" for premenopausal data in the forest plots.

\section{Results}

\section{Study selection}

Of 12,298 articles initially retrieved from all included databases, 3219 duplicate entries were automatically removed by the reference management software. Then, 7115 studies were excluded after title and abstract screening. The remaining 1964 studies were assessed according to the inclusion and exclusion criteria described above. As shown in the flow diagram (Fig. 1), a total of 10 studies were finally selected [30-39]. Details of study design, subject characteristics, and BMD outcomes are presented in Table 1. Detailed information about the exercise interventions can be found in Table 2 . The pooled number of participants was 668 (intervention group: $n=334$; control group: $n=334$ ) and sample size in individual studies ranged from 26 [30] to 97 participants [38]. The age of the participants ranged from 38 [36] to 77 years old [37], including therefore both premenopausal and postmenopausal women.

\section{Exercise interventions}

Three studies prescribed impact exercise such as jumping or skipping $[30,35,36]$. Four studies prescribed brisk walking [34, 37-39]. Tai Chi was employed in one study [33]. Unilateral standing on one leg was the chosen modality in one intervention [32]. Bodyweight exercises were utilized in one study [37] in combination with brisk walking. Another study used back extensions with external loading as exercise modality [31]. Intervention duration ranged from 6 months $[30,32]$ to 2 years $[31,37,38]$. The prescribed exercise frequency in the selected studies ranged from 3 sessions weekly
Fig. 1 Flow diagram of studies selection process according to the Preferred Reporting Items for Systematic Review and Meta-Analysis

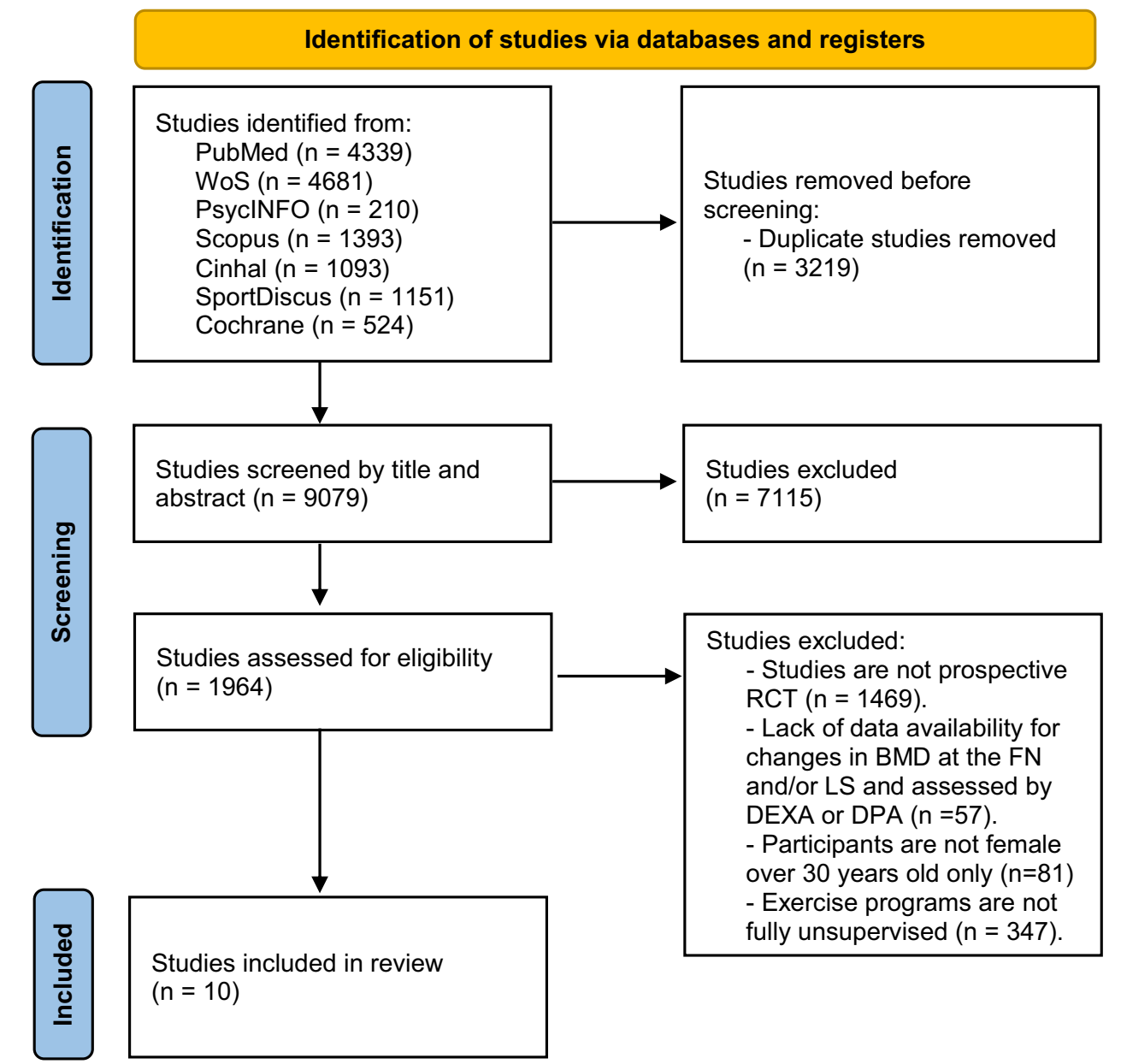


Table 1 Characteristics of selected studies

\begin{tabular}{|c|c|c|c|c|c|c|}
\hline Study & Mean age & $\begin{array}{l}N \\
\text { exercise } \\
\text { group }\end{array}$ & $\begin{array}{l}N \\
\text { control } \\
\text { group }\end{array}$ & Subjects characteristics & Dietary supplements & Outcome \\
\hline Greenway et al. (2015) & $\begin{array}{l}E: 41 \pm 8 \\
\text { C: } 41 \pm 8\end{array}$ & 36 & 55 & $\begin{array}{l}\text { Premenopausal with osteope- } \\
\text { nia/ osteoporosis }\end{array}$ & Calcium (1 g/day) & BMD at LS, FN \\
\hline Iwamoto et al. (2001) & $\begin{array}{l}E: 65 \pm 5 \\
C: 65 \pm 6\end{array}$ & 8 & 20 & $\begin{array}{l}\text { Postmenopausal with osteo- } \\
\text { porosis }\end{array}$ & $\begin{array}{l}\text { Calcium ( } 2 \mathrm{~g} / \text { day }) \text {, vitamin } \mathrm{D}_{3} \\
(1 \mu \mathrm{g} / \text { day })\end{array}$ & BMD at LS \\
\hline Niu et al. (2010) & $\begin{array}{l}\text { E: } 38 \pm 1 \\
\text { C: } 38 \pm 1\end{array}$ & 34 & 33 & Healthy premenopausal & Calcium (0.5 g/day) & $\mathrm{BMD}$ at $\mathrm{LS}, \mathrm{FN}$ \\
\hline Yamazaki et al. (2004) & $\begin{array}{l}E: 64 \pm 3 \\
C: 66 \pm 3\end{array}$ & 27 & 15 & $\begin{array}{l}\text { Postmenopausal with osteope- } \\
\text { nia/osteoporosis }\end{array}$ & Calcium (0.8 g/day) & BMD at LS \\
\hline Ebrahim et al. (1997) & $\begin{array}{l}\text { E: } 66 \pm 8 \\
\text { C: } 68 \pm 8\end{array}$ & 49 & 48 & $\begin{array}{l}\text { Postmenopausal who had frac- } \\
\text { tures in the previous } 2 \text { years }\end{array}$ & & BMD at LS, FN \\
\hline Brooke-Wavell et al. (1997) & $\begin{array}{l}\text { E: } 65 \pm 3 \\
\text { C: } 64 \pm 3\end{array}$ & 38 & 40 & Healthy postmenopausal & Calcium (0.85 g/day) & BMD at LS, FN \\
\hline Liu et al. (2015) & $\begin{array}{l}\text { E: } 63 \pm 7 \\
\text { C: } 62 \pm 8\end{array}$ & 48 & 42 & $\begin{array}{l}\text { Postmenopausal with osteo- } \\
\text { porosis }\end{array}$ & $\begin{array}{l}\text { Calcium (1.5 g/day), vitamin } \\
\mathrm{D}_{3}(15 \mu \mathrm{g} / \text { day })\end{array}$ & BMD at LS, FN \\
\hline Sakai et al. (2010) & $\begin{array}{l}\text { E: } 68 \pm 1 \\
\text { C: } 68 \pm 1\end{array}$ & 47 & 37 & Healthy postmenopausal & & $\mathrm{BMD}$ at FN \\
\hline Sugiyama et al. (2002) & $\begin{array}{l}\text { E: } 52 \pm 1 \\
\text { C: } 53 \pm 1\end{array}$ & 13 & 13 & $\begin{array}{l}\text { Healthy premenopausal and } \\
\text { postmenopausal }\end{array}$ & & BMD at LS, FN \\
\hline Sinaki et al. (1989) & $\begin{array}{l}\text { E: } 56 \pm 4 \\
\text { C: } 56 \pm 4\end{array}$ & 34 & 31 & Healthy postmenopausal & & BMD at LS \\
\hline
\end{tabular}

$E$ exercise group, $C$ control group, $L S$ lumbar spine, $F N$ femoral neck

$[30,36,38]$ to 3 sessions daily $[32,33]$. Further details can be found in Table 2 .

\section{Methods of delivery and of adherence assessment}

Different tools to administer and monitor these non-supervised programs were reported: filling exercise logs [35, 37, 39], following video-guided exercise sessions [36], heart-rate self-monitoring [34], or regular telephone calls [38]. The remaining studies included in this meta-analysis [30-33] do not describe the tools employed to deliver and monitor exercise programs.

\section{Attrition, adherence, and adverse effects}

Most of the selected studies reported data on attrition in their non-supervised exercise interventions: Brooke-Wavell et al. [39] report that 38 (out of 43) subjects in the intervention group and 40 (out of 41) in the control group completed the trial ( $12 \%$ and $2 \%$ dropout rate respectively), with dropouts due to reasons such as surgery, illness, or accident (not exercise-related). In the study by Ebrahim et al. [38], 49 (out of 81) subjects in the intervention group and 48 (out of 84) in the control group finished the intervention, registering therefore a substantial dropout rate $(40 \%$ and $42 \%$ respectively) due to unwillingness to continue and illness among the main reasons. Liu et al. [33] reported that 48 (out of 50) and 42 (out of 48) subjects in the intervention and control groups respectively completed the trial (4\% and $12 \%$ dropout rate), with reasons for withdrawal being serious back pain, traumatic fracture, and sudden heart attack unrelated to the study. Niu et al. [36] informed a dropout rate of $24 \%$ in the intervention group and $28 \%$ in the control group, with 34 (out of 45) and 33 (out of 46) subjects completing the trial, respectively. In the study by Sakai et al. [32], the number of subjects who completed the study was 47 (out of 49) for the intervention group and 37 (out of 45) for the control group (4\% and $12 \%$ dropout rate respectively). Sixty-five (out of 68) subjects completed the study by Sinaki et al. [31] (4\% dropout rate), with all three withdrawals happening in the control group for reasons such as moving to a different state or illness. Finally, Yamazaki et al. [34] reported that 42 (out of 50) subjects completed the trial (16\% dropout rate). Therefore, the dropout rate ranged from 0 to $40 \%$ in the exercise groups (mean $\pm \mathrm{SD}=14.2 \% \pm 13.9 \%)$ and 2.4 to $42 \%$ in the control groups (mean $\pm \mathrm{SD}=18.4 \% \pm 13.4 \%$ ).

Adherence to the exercise programs was reported by most of the studies included in this meta-analysis. Thus, Greenway et al. [35] reported a mean compliance of 53\% of prescribed sessions (range 20-90\%). Niu et al. [36] describes that the median number of exercise sessions attended was 2.4 times per week. The study by Ebrahim et al. [38] informs that all the women who remained in the intervention reported carrying out the prescribed exercise sessions. Brooke-Wavell 
Table 2 Exercise interventions

\begin{tabular}{|c|c|c|c|c|}
\hline Study & Exercise category & Intervention duration & $\begin{array}{l}\text { Frequency } \\
\text { (sessions per } \\
\text { week) }\end{array}$ & Intervention description \\
\hline Greenway et al. (2015) & DWBHF & 15 months & 4 & $\begin{array}{l}\text { Unilateral landings after walking off a raised step. } \\
\text { Step height progressed from } 18.5 \text { to } 63.5 \mathrm{~cm} \text { (incre- } \\
\text { ments of } 7.5 \text { or } 5 \mathrm{~cm} \text { every } 8 \text { weeks). Landings were } \\
\text { divided into } 2 \text { to } 5 \text { sets per foot, ranging from } 3 \text { to } \\
10 \text { repetitions per set. Total number of landings per } \\
\text { session started at } 12 \text { and progressed to } 80 \text {, where } \\
\text { it remained constant from week } 16 \text {. Landing foot } \\
\text { alternated after every set }\end{array}$ \\
\hline Iwamoto et al. (2001) & COMB & 24 months & 7 & $\begin{array}{l}\text { Walking: Subjects were encouraged to increase their } \\
\text { baseline daily step count by } 30 \% \text { thorough brisk } \\
\text { walking } \\
\text { Gymnastic training: } 2 \text { sets } \times 15 \text { repetitions of straight } \\
\text { leg raising, squatting, and abdominal and back } \\
\text { muscle exercises }\end{array}$ \\
\hline Niu et al. (2010) & DWBHF & 12 months & $\geq 3$ & $\begin{array}{l}\text { Each session included a } 3 \text {-min warm-up (stretching), } \\
5 \text { sets } \times 10 \text { countermovement jumps, and a } 3 \text {-min } \\
\text { cooldown (stretching) }\end{array}$ \\
\hline Yamazaki et al. (2004) & DWBLF & 12 months & 4 & $\begin{array}{l}\text { Outdoor walking at a speed corresponding to } 50 \% \text { of } \\
\text { the maximal oxygen consumption, a minimum of } \\
8000 \text { steps per session }\end{array}$ \\
\hline Ebrahim et al. (1997) & DWBLF & 24 months & 3 & $\begin{array}{l}\text { Self-paced brisk walking for } 40 \mathrm{~min} \text { per session. } \\
\text { Subjects were instructed to walk at a faster pace than } \\
\text { usual but avoiding discomfort or shortness of breath }\end{array}$ \\
\hline Brooke-Wavell et al. (1997) & DWBLF & 12 months & 7 & Self-paced brisk walking, 280 min per week \\
\hline Liu et al. (2015) & DWBLF & 12 months & 21 ( $3 \times$ daily $)$ & $\begin{array}{l}\text { Tai-Chi sessions consisting of } 7 \text { repetitions of the } \\
\text { "modified eighth section of Eight-Section Brocade." }\end{array}$ \\
\hline Sakai et al. (2010) & SWB & 6 months & 21 ( $3 \times$ daily) & $\begin{array}{l}\text { Unilateral standing exercise: each session consisted of } \\
\text { standing on each leg for } 1 \mathrm{~min}\end{array}$ \\
\hline Sugiyama et al. (2002) & DWBHF & 6 months & 3 & Rope skipping, 100 jumps/session \\
\hline Sinaki et al. (1989) & NWBLF & 24 months & 5 & $\begin{array}{l}\text { Back extensions in prone position with backpack con- } \\
\text { taining weights equivalent to } 30 \% \text { of each subject's } \\
\text { maximal isometric back muscle strength, } 10 \text { repeti- } \\
\text { tions per session }\end{array}$ \\
\hline
\end{tabular}

$E$ exercise group, $C$ control group, $S W B$ static weight-bearing, $D W B L F$ dynamic weight-bearing exercise low force, $D W B H F$ Dynamic weightbearing exercise high force, $N W B L F$ non-weight-bearing exercise low force, $C O M B$ combination.

et al. [39] report that participants completed, on average, $20.4 \mathrm{~min} /$ day, during an average of 4.8 walks per week, out of the 7 weekly sessions prescribed. Liu et al. [33] inform that average compliance was $96 \%$, while Sakai et al. [32] describe a $71.4 \%$ compliance to prescribed sessions. Finally, in the study by Sugiyama et al. [30], an average compliance of $75 \%$ is reported among premenopausal women, and $71.4 \%$ among postmenopausal women. In summary, adherence to the non-supervised exercise programs ranged from 53 to $100 \%$ (mean $\pm \mathrm{SD}=78.3 \% \pm 16.1 \%$ ).

Regarding the safety of non-supervised exercise interventions, the selected studies reported the following injuries and adverse effects: Brooke-Wavell et al. [39] report that two women had minor foot problems. Ebrahim et al. [38] reported 71 falls in the intervention group and 54 falls in the control group, as well as 6 fractures in the intervention group and 4 fractures in the control group during the 2-year period. Finally, Greenway et al. [35] specified that no injuries occur during their intervention, while Liu et al. [33] reported no serious injuries or adverse events associated with exercises, and four minor injuries related to knee, back, or hip pain.

\section{Methodology quality}

PEDro scores are shown in Table 3. The methodological quality of one study is classified as high [38], seven studies demonstrated moderate [31-33, 35-37, 39], and the remaining two studies are classified as being of low methodological quality [30, 34]. 


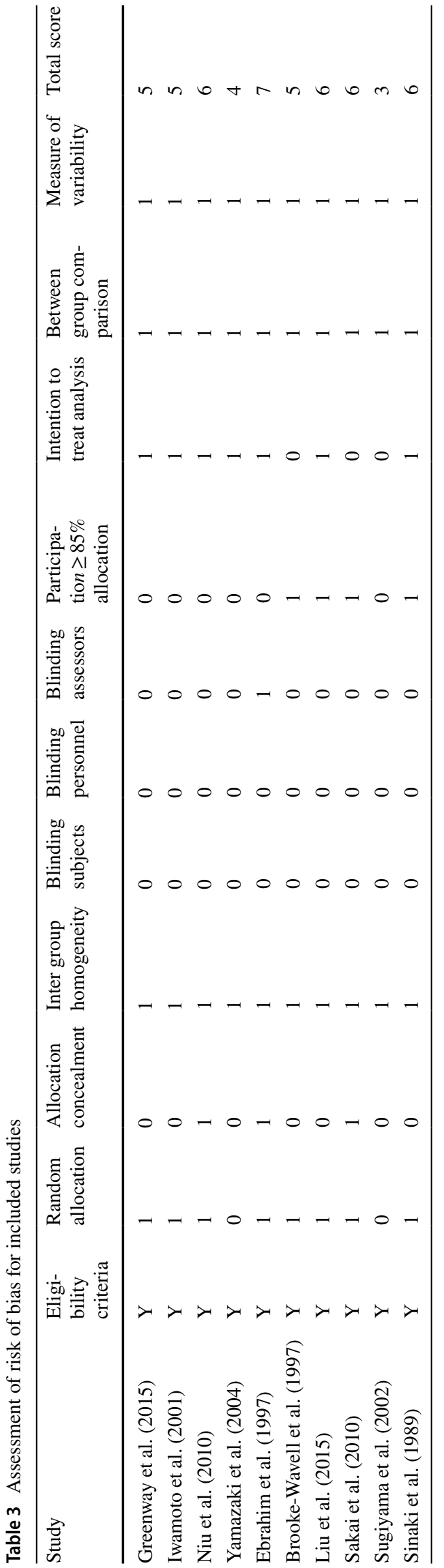

\section{Meta-analysis results}

The following meta-analyses were performed to investigate the effect of non-supervised exercise on BMD. BMD was measured by DXA in 9 of the selected studies [30, 32-39], and by DPA in one of the selected studies [31]. Nine of the studies reported BMD at LS [30, 31, 33-39], and seven studies reported BMD at FN [30, 32, 33, 35, 36, 38, 39].

\section{Effect of non-supervised exercise on BMD at the LS}

Nine studies assessed the effect of non-supervised exercise interventions on BMD at the LS. Random effect analysis showed that non-supervised exercise significantly increased LS BMD relative to control (SMD: 0.40, 95\% CI: 0.03-0.77, $P<0.05)$. Heterogeneity between studies was substantial $\left(I^{2}=79 \%, Q=41.93\right.$, degrees of freedom $=9, P<0.00001$; Fig. 2). A sensitivity analysis omitting the two studies with lowest methodological quality [30,34] revealed likewise significant positive effects of non-supervised exercise on BMD at the LS (SMD: 0.55, 95\% CI: 0.09-1.00, $P<0.05$ ).

\section{Effect of non-supervised exercise on BMD at the FN}

Seven studies assessed the effect of non-supervised exercise on BMD at the FN. Random effect analysis showed that nonsupervised exercise significantly increased FN BMD relative to control (SMD: 0.51, 95\% CI: 0.16-0.85, $P<0.05$ ). Heterogeneity between studies was substantial $\left(I^{2}=74 \%\right.$, $Q=27.26$, degrees of freedom $=7, P=0.0003$; Fig. 3). A sensitivity analysis omitting the study with lowest methodological quality [30] revealed likewise significant positive effects of non-supervised exercise on BMD at the FN (SMD: 0.48, 95\% CI: $0.08-0.88, P<0.05)$.

Replacing correlation coefficients with 0.7 in sensitivity analyses did not affect the findings of this study.

\section{Subgroup analyses}

Subgroup analyses were performed to determine the effect of menopause and osteoporosis/osteopenia status, trial duration, and exercise mode on exercise-related BMD changes. Results are summarized in Table 4.

\section{Menopausal status}

Similar non-significant effects on LS BMD were found in premenopausal $(\mathrm{SMD}=0.48 ; 95 \% \mathrm{CI}:-0.44,1.41)$ and postmenopausal women $(\mathrm{SMD}=0.36,95 \% \mathrm{CI}:-0.04$, $0.75)$, with no significant differences between subgroups according to random effects analysis $(P=0.81$; Supplementary Fig. 1). Non-supervised exercise improved FN BMD in premenopausal women $(\mathrm{SMD}=0.76 ; 95 \%$ 


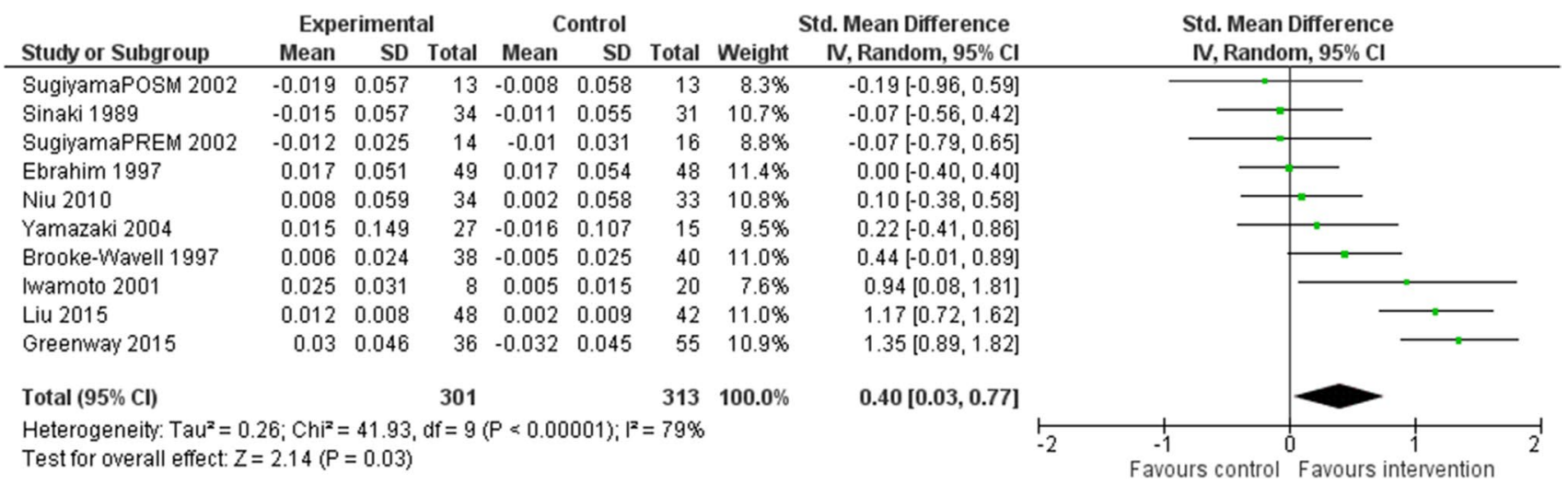

Fig. 2 Forest plot of meta-analysis results on BMD at the LS

\begin{tabular}{|c|c|c|c|c|c|c|c|}
\hline \multirow[b]{2}{*}{ Study or Subgroup } & \multicolumn{3}{|c|}{ Experimental } & \multicolumn{3}{|c|}{ Control } & \multirow[b]{2}{*}{ Weight } \\
\hline & Mean & SD & Total & Mean & SD & Total & \\
\hline Sakai 2010 & -0.001 & 0.052 & 47 & 0.001 & 0.043 & 37 & $13.7 \%$ \\
\hline Brooke-Wavell 1997 & 0.016 & 0.037 & 38 & 0.011 & 0.044 & 40 & $13.5 \%$ \\
\hline Niu 2010 & 0.004 & 0.061 & 34 & -0.008 & 0.04 & 33 & $13.0 \%$ \\
\hline SugiyamaPOSM 2002 & 0.005 & 0.067 & 13 & -0.008 & 0.024 & 13 & $9.3 \%$ \\
\hline Ebrahim 1997 & -0.002 & 0.042 & 49 & -0.021 & 0.065 & 48 & $14.1 \%$ \\
\hline SugiyamaPREM 2002 & 0.018 & 0.025 & 14 & -0.006 & 0.021 & 16 & $9.4 \%$ \\
\hline Greenway 2015 & 0.018 & 0.037 & 36 & -0.023 & 0.038 & 55 & $13.5 \%$ \\
\hline Liu 2015 & 0.007 & 0.008 & 48 & -0.006 & 0.014 & 42 & $13.5 \%$ \\
\hline Total $(95 \% \mathrm{Cl})$ & & & 279 & & & 284 & $100.0 \%$ \\
\hline
\end{tabular}

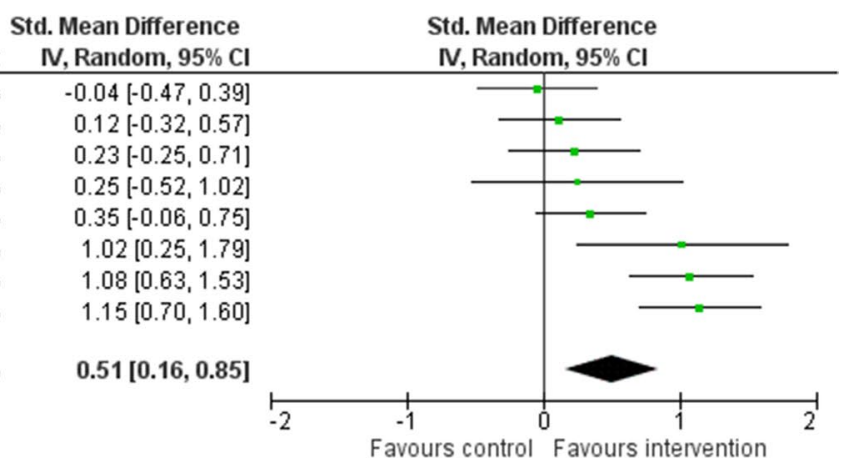

Fig. 3 Forest plot of meta-analysis results on BMD at the FN

CI: $0.16,1.35)$, but not in postmenopausal women $(\mathrm{SMD}=0.37 ; 95 \% \mathrm{CI}:-0.06,0.80)$. However, there were no differences between subgroups according to random effects analysis ( $P=0.30$; Supplementary Fig. 2).

\section{Trial duration}

Similar non-significant effects on LS BMD were found in trials with duration $\leq 1$ year $(\mathrm{SMD}=0.33 ; 95 \% \mathrm{CI}:-0.09$, $0.75)$ and trials with duration $>1$ year $(\mathrm{SMD}=0.53 ; 95 \%$ CI: $-0.21,1.28$ ), with no significant differences between subgroups according to random effects analysis $(P=0.63$; Supplementary Fig. 3). Non-supervised exercise improved FN BMD in trials that went for a duration of 1 year or less $(\mathrm{SMD}=0.44 ; 95 \% \mathrm{CI}: 0.01,0.86)$, but not in trials that exceeded 1 year in duration $(\mathrm{SMD}=0.71 ; 95 \% \mathrm{CI}:-0.02$, 1.43). However, there were no differences between subgroups according to random effects analysis $(P=0.53$; Supplementary Fig. 4).

\section{Exercise modality}

Non-significant effects on LS BMD were found for DWBLF (SMD =0.46; 95\% CI: $-0.07,1.00)$ and DWBHF $(\mathrm{SMD}=0.33 ; 95 \% \mathrm{CI}:-0.45,1.11)$. There were no differences between subgroups according to random effects analysis $(P=0.78$; Supplementary Fig. 5). Non-significant effects on FN BMD were found for DWBLF (SMD = 0.54; 95\% CI: $-0.06,1.13)$. DWBHF had a significant positive effect on FN BMD (SMD $=0.65 ; 95 \%$ CI: $0.16,1.15)$. There were no differences between subgroups according to random effects analysis ( $P=0.77$; Supplementary Fig. 6).

\section{Osteopenia/osteoporosis status}

A subgroup analysis was performed to assess whether the effect of exercise on BMD is dependent on bone health status. Thus, in this subgroup analysis, BMD adaptations between women with osteopenia or osteoporosis [33-35, 37, 38] and healthy women (i.e., women with no diagnosed bone disease) 
Table 4 Summary of subgroup analyses

\begin{tabular}{|c|c|c|c|c|c|c|c|c|}
\hline Subgroup & $N(\mathrm{~K})$ & $\operatorname{SMD}(95 \% \mathrm{CI})$ & $P($ Z-test $)$ & $\tau^{2}$ & $Q$ statistic & $I^{2}$ & $P\left(\chi^{2}\right.$ test $)$ & $\begin{array}{l}P \text { (subgroup } \\
\text { differences) }\end{array}$ \\
\hline \multicolumn{9}{|l|}{ LS BMD } \\
\hline \multicolumn{9}{|l|}{ Menopausal status } \\
\hline -Premenopausal & $188(3)$ & $0.48(-0.44,1.41)$ & 0.31 & 0.59 & 17.56 & $89 \%$ & 0.0002 & \multirow[t]{2}{*}{0.81} \\
\hline -Postmenopausal & $426(7)$ & $0.36(-0.04,0.75)$ & 0.08 & 0.20 & 22.59 & $73 \%$ & 0.0009 & \\
\hline \multicolumn{9}{|l|}{ Trial duration } \\
\hline$-\leq 1$ year & $333(6)$ & $0.33(-0.09,0.75)$ & 0.13 & 0.19 & 16.90 & $70 \%$ & 0.005 & \multirow[t]{2}{*}{0.63} \\
\hline$->1$ year & $281(4)$ & $0.53(-0.21,1.28)$ & 0.16 & 0.49 & 25.02 & $88 \%$ & $<0.0001$ & \\
\hline \multicolumn{9}{|l|}{ Exercise modality } \\
\hline -DWBLF & $307(4)$ & $0.46(-0.07,1.00)$ & 0.09 & 0.23 & 15.23 & $80 \%$ & 0.002 & \multirow[t]{2}{*}{0.78} \\
\hline -DWBHF & $214(4)$ & $0.33(-0.45,1.11)$ & 0.40 & 0.53 & 21.02 & $86 \%$ & 0.0001 & \\
\hline \multicolumn{9}{|l|}{ Osteopenia/osteoporosis status } \\
\hline -Healthy women & $266(5)$ & $0.11(-0.13,0.35)$ & 0.37 & 0.00 & 3.45 & $0 \%$ & 0.49 & \multirow[t]{2}{*}{0.06} \\
\hline -Women with osteopenia/ osteoporosis & $348(5)$ & $0.73(0.13,1.33)$ & 0.02 & 0.38 & 26.12 & $85 \%$ & $<0.0001$ & \\
\hline \multicolumn{9}{|l|}{ FN BMD } \\
\hline \multicolumn{9}{|l|}{ Menopausal status } \\
\hline -Premenopausal & $188(3)$ & $0.76(0.16,1.35)$ & 0.01 & 0.19 & 7.05 & $72 \%$ & $\mathbf{0 . 0 3}$ & \multirow[t]{2}{*}{0.30} \\
\hline -Postmenopausal & $375(5)$ & $0.37(-0.06,0.80)$ & 0.09 & 0.18 & 16.45 & $76 \%$ & 0.002 & \\
\hline \multicolumn{9}{|l|}{ Trial duration } \\
\hline$-\leq 1$ year & $375(6)$ & $0.44(0.01,0.86)$ & 0.04 & 0.20 & 19.41 & $74 \%$ & 0.002 & \multirow[t]{2}{*}{0.53} \\
\hline$->1$ year & $188(2)$ & $0.71(-0.02,1.43)$ & 0.05 & 0.22 & 5.72 & $82 \%$ & 0.02 & \\
\hline \multicolumn{9}{|l|}{ Exercise modality } \\
\hline -DWBLF & $265(3)$ & $0.54(-0.06,1.13)$ & 0.08 & 0.23 & 11.41 & $82 \%$ & 0.003 & \multirow[t]{2}{*}{0.77} \\
\hline -DWBHF & $214(4)$ & $0.65(0.16,1.15)$ & 0.01 & 0.16 & 8.36 & $64 \%$ & 0.04 & \\
\hline \multicolumn{9}{|l|}{ Osteopenia/osteoporosis status } \\
\hline -Healthy women & $285(5)$ & $0.22(-0.77,0.51)$ & 0.13 & 0.03 & 5.70 & $30 \%$ & 0.22 & \multirow[t]{2}{*}{0.04} \\
\hline -Women with osteopenia/ osteoporosis & $278(3)$ & $0.85(0.33,1.37)$ & 0.001 & 0.16 & 8.76 & $77 \%$ & 0.01 & \\
\hline
\end{tabular}

Data in boldface type indicate significance. $N$ number of subjects, $K$ number of studies, $S M D$ standardized mean difference, $C I$ confidence interval, $B M D$ bone mineral density, $D W B L F$ dynamic weight-bearing exercise low force, $D W B H F$ dynamic weight-bearing exercise high force. Not enough studies to compute heterogeneity.

[30, 31, 36, 39] were compared. A significant positive effect on LS BMD was found for women with osteopenia or osteoporosis $(\mathrm{SMD}=0.73 ; 95 \% \mathrm{CI}: 0.13,1.33)$, while effects were nonsignificant in healthy women $(\mathrm{SMD}=0.11 ; 95 \% \mathrm{CI}:-0.13$, 0.35). However, there were no differences between subgroups according to random effects analysis ( $P=0.06$; Supplementary Fig. 7). A significant positive effect on FN BMD was found for women with osteopenia or osteoporosis $(\mathrm{SMD}=0.85 ; 95 \%$ CI: $0.33,1.37$, while effects were non-significant in healthy women $(\mathrm{SMD}=0.22 ; 95 \% \mathrm{CI}:-0.07,0.51)$. Differences between subgroups were significant according to random effects analysis ( $P=0.04$; Supplementary Fig. 8).

\section{Discussion}

The main aim of this study was to assess the effectiveness of non-supervised exercise programs for bone health via a systematic review and meta-analysis of RCTs. Non-supervised exercise programs improved BMD at the FN and LS in adult women. An intervention based on DWBHF (e.g., jumping, plyometrics) could be most beneficial on FN BMD. The positive effects of non-supervised exercise on BMD might be observable in less than 1 year and more pronounced in women with osteopenia or osteoporosis.

Our study reveals that non-supervised, home-based exercise programs can be an effective alternative to supervised exercise. More specifically, our findings show that nonsupervised exercise programs can be effective at significantly improving BMD at the LS and FN in adult women. Moreover, the effect size found in the current study (SMD$\mathrm{LS}=0.40$, SMD-FN $=0.51$ ) was comparable to a recent meta-analysis that included both supervised and non-supervised exercise interventions [12] (SMD-LS =0.37, SMD$\mathrm{FN}=0.33$ ). In order to make our results more clinically relevant, standardized mean differences between intervention and control groups were converted to areal BMD units, finding a value of $0.015 \mathrm{~g} / \mathrm{cm}^{2}(95 \% \mathrm{CI}: 0.004-0.026)$ at 
FN and $0.013 \mathrm{~g} / \mathrm{cm}^{2}$ (95\% CI: 0.002-0.024) at LS. Looking specifically at women with osteopenia/osteoporosis, mean differences are greater: $0.024 \mathrm{~g} / \mathrm{cm}^{2}$ (95\% CI: 0.005-0.042) at FN and $0.023 \mathrm{~g} / \mathrm{cm}^{2}$ (95\% CI: 0.001-0.046) at LS. This mean difference in BMD between groups equates to $3.14 \%$ at the FN and $2.88 \%$ at the LS. According to recent studies, these BMD improvements reduce fracture risk by an estimated $35 \%, 25 \%$, and $15 \%$ in vertebral, hip, and nonvertebral fractures respectively [40-42]. Indeed, epidemiological evidence suggests that exercise significantly reduces the risk of osteoporotic fractures, probably through a combined effect of improved BMD, muscle strength, flexibility, and balance [43].

Non-supervised exercise improved BMD at the FN in studies with intervention durations of $<1$ year, but it had no effect on BMD in studies with intervention durations of $>1$ year at both the FN and LS. This result is inconsistent with other studies showing that longer exposure to exercise generally results in better bone health [44]. Longer exercise interventions are required to observe bone adaptations and physiological changes in BMD, at least when there is an adequate progression of the exercise load [6]. This required progression might have been insufficient in the four selected studies that had trial durations over 1 year: two of them maintained the exercise load constant throughout the intervention [31,37], another study just instructed the participants to progressively increase the amount and speed of walking "in a manner that suited them" [38], and only one of the selected studies with a trial duration of over 1 year designed a protocol to progressively increase the exercise load [35], obtaining the highest size effect within the subgroup (see Supplementary Figs. 3 and 4). Therefore, it could be argued that longer interventions can only elicit better effects if they are paired with an adequate progression in exercise loads. If consistent progression is missing, further BMD changes after initial adaptations may be hindered, due to non-compliance with the overload principle [45]. Bone tissue must be stimulated with loads exceeding a certain threshold to elicit positive bone adaptations [46]. Therefore, it has been suggested that bone-loading forces should be objectively quantified and monitored to ensure the efficacy of interventions for osteoporosis prevention or treatment [47]. New technologies such as accelerometers could assist in the implementation of effective non-supervised interventions to improve bone health [10].

Exercise modality is a key factor to achieve sufficient mechanical forces. The type of exercise and the forces it generates might be the most critical determinants of the effect of exercise on bone [48]. In this respect, the current official guidelines on exercise prescription for the prevention and management of osteoporosis underscore the efficacy of impact activities and high-intensity progressive resistance training [6]. The subgroup analysis performed in the current study to analyze differences in BMD changes between exercise modalities found significant effects for DWBHF (e.g., jumping) at the $\mathrm{FN}$, as well as a trend towards significance at both skeletal sites for DWBHL (e.g., brisk walking) (see Supplementary Figs. 5 and 6). Combining resistance and impact training to improve BMD at the FN and LS has produced very positive results in the LIFTMOR trial, which prescribed a high-intensity resistance and impact exercise intervention in postmenopausal women with low bone mass [49]. These results are consistent with the outcomes of recent meta-analyses, where a combination of resistance training with impact training appears to elicit the highest osteogenic response at the spine and hip [6,50]. Moreover, these results echo findings from two recent studies from two independent cohorts of masters athletes. Masters athletes are people who continue to train for and compete in athletic events beyond their 4 th, and often into their 8 th and 9 th decade of life [51]. A 10-year follow-up of 69 male sprinters revealed maintained or improved tibia bone strength for athletes who continued to train, but maintenance or loss in those who reduced or abandoned regular sprint training [52]. An independent cohort of 71 track-and-field masters athletes with mean followed-up period of 4.2 years confirms improvements in tibial bone strength over time in male power athletes, with maintenance of bone strength at best in female power athletes, and with age-related bone losses in endurance athletes of either sex [53]. These findings are remarkable in two ways. Firstly, they highlight the efficacy of certain track-and-field-related training modes (strength, plyometric and high-intensity interval training) for maintaining or improving BMD. Secondly, these two studies suggest that intrinsic motivation and positive affect, which are intrinsically high in masters athletes [54], may be instrumental allies when trying to enhance acceptance of and adherence to exercise programs. Other studies also support the benefits of multi-modal programs that incorporate not only osteoanabolic exercises, but also activities aimed to develop balance and proprioception to prevent falls $[55,56]$.

The effect of non-supervised exercise on BMD at different skeletal sites did not differ in premenopausal and postmenopausal women (see Supplementary Figs. 1 and 2). As bone loss increases rapidly after menopause [57], osteoporosis prevention programs to attain and maintain healthy bones in young premenopausal women are critical to reduce the risk or at least delay the development of the disease in later life $[58,59]$. Despite these findings, BMD responses to exercise appear to be greater in women with osteopenia/osteoporosis compared to healthy counterparts. Our subgroup analysis showed a greater positive effect on BMD at both the LS and FN for women with osteopenia or osteoporosis (SMD-LS $=0.73$, SMD-FN $=0.85$ ) than for healthy women (SMD-LS $=0.11, \mathrm{SMD}-\mathrm{FN}=0.22$ ). This suggests that exercise interventions will be most beneficial 
for women who already start with an impaired bone health status. Different factors could mediate this increased adaptation of exercise in women with poorer bone health, such as enhanced physiological response in weaker bones [60], or a higher commitment and adherence to exercise among women who have been diagnosed with such a condition [61].

A low dropout rate was generally reported in the selected studies. Specifically, the percentages of participants in the intervention groups completing the whole study ranged from $53[38]$ to $96 \%[32,33]$. Three of the included studies [30, $35,37]$ did not report any information regarding attrition. No major differences were observed between intervention and control groups, since average attrition rate was $14.2 \%$ and $18.4 \%$ respectively. A meta-analysis assessing dropouts in exercise interventions targeting BMD in adults reported similar attrition rates in supervised exercise interventions ( $21 \%$ for the intervention groups and $16 \%$ for the control groups), and found no statistically significant differences between non-supervised versus supervised exercise, or a combination of supervised and non-supervised exercise [13].

Average compliance to the prescribed exercise sessions was $78.3 \%$ in the included studies. These results are similar to previous studies. In a recent meta-analysis, average attendance rate was $82 \%$ for supervised interventions, and $75 \%$ for non-supervised interventions [12]. As mentioned above, it is possible that home-based non-supervised training increases adherence by overcoming barriers such as lack of time and difficulties with transportation [14, 15]. On the other hand, fear of falling and safety concerns might decrease adherence to non-supervised intervention for older adults [62]; in fact, one of the studies included in this metaanalysis, Ebrahim et al. [38], reported a higher number of falls and fractures in the intervention group compared with the control. Some factors suggested in the literature to improve adherence in home-based interventions include social or familiar support, and a reduced number of exercises in the program [63]. The use of new technologies to monitor and guide non-supervised training may also have a positive effect on exercise adherence in older adults [64, 65]. It would be of interest to develop intervention programs in which technologies are used to monitor intensity, provide tailored guidelines, and implement an adequate progression while allowing subjects to carry out the training sessions at home without direct supervision.

There are limitations that could reduce the validity of this systematic review and meta-analysis. First, there were notable variations between effect sizes across the selected studies, raising questions about the importance of confounding variables in the included studies (e.g., diet), or dissimilar methodologies (e.g., precision of outcome measurements) when attempting to aggregate data for meta-analysis [66]. This study has focused on LS and FN BMD; it would be of interest to extend this analysis to other sites, such as the radius or total hip. Another limitation in our approach is that, due to the reduced number of studies meeting the inclusion criteria, sub-analyses were likely under powered to detect significance differences in BMD changes between groups. Our analyses were also performed on aggregate data, not individual patient data. Despite such limitations, the findings in this study demonstrate that non-supervised exercise is an effective intervention for improving BMD in adult women. Further research is required to evaluate the contribution of single exercise variables (e.g., intensity, volume, density) on bone health that may illuminate even more efficient recommendations.

\section{Conclusion}

Non-supervised exercise programs improve BMD at FN and LS in adult women. Interventions based on DWBHF (e.g., jumping, plyometrics) might be most effective for improving FN BMD in adult women. Positive effects of non-supervised exercise on BMD might be observed in periods shorter than a year and especially effective in women already diagnosed with osteopenia or osteoporosis. Due to the low number of trials conducted to date, more RCTs prescribing non-supervised, osteogenic exercise are required in adult women. More trials investigating the efficacy of remote/assistive technologies for delivering and monitoring non-supervised exercise interventions are also necessary.

Supplementary Information The online version contains supplementary material available at https://doi.org/10.1007/s00198-022-06357-3.

Funding This work was supported by the Ministry of Universities (Government of Spain). Grant FPU19/00991.

Data availability Available upon request.

Code availability Not applicable.

\section{Declarations}

Conflicts of interest None.

\section{References}

1. Kanis JA, Melton LJ III, Christiansen C, Johnston CC, Khaltaev N (1994) The diagnosis of osteoporosis. J Bone Miner Res 9:1137-1141

2. Wright NC, Looker AC, Saag KG, Curtis JR, Delzell ES, Randall S, Dawson-Hughes B (2014) The recent prevalence of osteoporosis and low bone mass in the United States based on bone mineral density at the femoral neck or lumbar spine. J Bone Miner Res 29:2520-2526

3. Sozen T, Ozisik L, Basaran NC (2017) An overview and management of osteoporosis. Eur J Rheumatol 4:46-56 
4. Hernlund E, Svedbom A, Ivergard M, Compston J, Cooper C, Stenmark J, McCloskey EV, Jonsson B, Kanis JA (2013) Osteoporosis in the European Union: medical management epidemiology and economic burden A report prepared in collaboration with the International Osteoporosis Foundation (IOF) and the European Federation of Pharmaceutical Industry Associations (EFPIA). Archives Osteoporosis 8:136

5. Compston J (2010) Osteoporosis: social and economic impact. Radiol Clin North Am 48:477-482

6. Beck BR, Daly RM, Singh MAF, Taaffe DR (2017) Exercise and Sports Science Australia (ESSA) position statement on exercise prescription for the prevention and management of osteoporosis. J Sci Med Sport 20:438-445

7. Tarantino U, Iolascon G, Cianferotti L, Masi L, Marcucci G, Giusti F, Marini F, Parri S, Feola M, Rao C (2017) Clinical guidelines for the prevention and treatment of osteoporosis: summary statements and recommendations from the Italian Society for Orthopaedics and Traumatology. J Orthop Traumatol 18:3-36

8. Wolff I, van Croonenborg JJ, Kemper HCG, Kostense PJ, Twisk JWR (1999) The effect of exercise training programs on bone mass: a meta-analysis of published controlled trials in pre- and postmenopausal women. Osteoporos Int 9:1-12

9. Frost HM (1988) Vital biomechanics: proposed general concepts for skeletal adaptations to mechanical usage. Calcif Tissue Int 42:145-156

10. Sañudo B, de Hoyo M, del Pozo-Cruz J, Carrasco L, del PozoCruz B, Tejero S, Firth E (2017) A systematic review of the exercise effect on bone health: the importance of assessing mechanical loading in perimenopausal and postmenopausal women. Menopause 24:1208-1216

11. Ng C-A, McMillan L, Humbert L, Ebeling P, Scott D (2020) Feasibility, safety and effectiveness of a pilot 16-week home-based, impact exercise intervention in postmenopausal women with low bone mineral density. Osteoporosis International 1-13

12. Shojaa M, Von Stengel S, Schoene D, Kohl M, Barone G, Bragonzoni L, Dallolio L, Marini S, Murphy MH, Stephenson A (2020) Effect of exercise training on bone mineral density in post-menopausal women: a systematic review and meta-analysis of intervention studies. Frontiers in Physiology 11:

13. Kelley GA, Kelley KS (2013) Dropouts and compliance in exercise interventions targeting bone mineral density in adults: a metaanalysis of randomized controlled trials. Journal of osteoporosis 2013:

14. Rodrigues I, Armstrong J, Adachi J, MacDermid J (2017) Facilitators and barriers to exercise adherence in patients with osteopenia and osteoporosis: a systematic review. Osteoporos Int 28:735-745

15. Simek EM, McPhate L, Hill KD, Finch CF, Day L, Haines TP (2015) What are the characteristics of home exercise programs that older adults prefer?: A cross-sectional study. Am J Phys Med Rehabil 94:508-521

16. Khan W (2020) Coronavirus disease 2019 (covid-19). Journal of Saidu Medical College, Swat 10:

17. Hammami A, Harrabi B, Mohr M, Krustrup P (2020) Physical activity and coronavirus disease 2019 (COVID-19): specific recommendations for home-based physical training. Managing Sport and Leisure 1-6

18. Stockwell S, Trott M, Tully M, Shin J, Barnett Y, Butler L, McDermott D, Schuch F, Smith L (2021) Changes in physical activity and sedentary behaviours from before to during the COVID-19 pandemic lockdown: a systematic review. BMJ Open Sport \& Exercise Medicine 7:e000960

19. Pinelli E, Barone G, Marini S, Benvenuti F, Murphy MH, Julin M, Kemmler W, Von Stengel S, Di Paolo S, Dallolio L (2021) Effects of COVID-19 lockdown on adherence to individual home-or gymbased exercise training among women with postmenopausal osteoporosis. Int J Environ Res Public Health 18:2441
20. Page MJ, McKenzie JE, Bossuyt PM, Boutron I, Hoffmann TC, Mulrow CD, Shamseer L, Tetzlaff JM, Akl EA, Brennan SE (2021) The PRISMA 2020 statement: an updated guideline for reporting systematic reviews. Bmj 372:

21. Unnanuntana A, Rebolledo BJ, Khair MM, DiCarlo EF, Lane JM (2011) Diseases affecting bone quality: beyond osteoporosis. Clin Orthop Relat Res 469:2194-2206

22. Del Rey FJA, González OP (2018) Epidemiology of osteoporotic fractures in Andalusia, Spain, from 2000-2010. Medicina Clínica (English Edition) 150:297-302

23. Howe TE, Shea B, Dawson LJ, Downie F, Murray A, Ross C, Harbour RT, Caldwell LM, Creed G (2011) Exercise for preventing and treating osteoporosis in postmenopausal women. Cochrane database of systematic reviews

24. Sherrington C, Herbert R, Maher C, Moseley A (2000) PEDro. A database of randomized trials and systematic reviews in physiotherapy. Man Ther 5:223-226

25. Ribeiro de Ávila V, Bento T, Gomes W, Leitão J, de Sousa NF (2018) Functional outcomes and quality of life after ankle fracture surgically treated: a systematic review. J Sport Rehabil 27:274-283

26. Borenstein M, Hedges LV, Higgins JP, Rothstein HR (2011) Introduction to meta-analysis. John Wiley \& Sons

27. Higgins JP, Thomas J, Chandler J, Cumpston M, Li T, Page MJ, Welch VA (2019) Cochrane handbook for systematic reviews of interventions. John Wiley \& Sons

28. Cohen J (2013) Statistical power analysis for the behavioral sciences. Academic press,

29. Deeks J, Higgins J, Altman D (2006) Analysing and presenting results. Cochrane Handbook Syst Rev Interventions 4:97-166

30. Sugiyama T, Yamaguchi A, Kawai S (2002) Effects of skeletal loading on bone mass and compensation mechanism in bone: a new insight into the "mechanostat" theory. J Bone Miner Metab 20:196-200

31. Sinaki M, Wahner HW, Offord KP, Hodgson SF (1989) Efficacy of nonloading exercises in prevention of vertebral bone loss in postmenopausal women: a controlled trial. Mayo Clin Proc 64:762-769

32. Sakai A, Oshige T, Zenke Y, Yamanaka Y, Nagaishi H, Nakamura $\mathrm{T}$ (2010) Unipedal standing exercise and hip bone mineral density in postmenopausal women: a randomized controlled trial. J Bone Miner Metab 28:42-48

33. Liu BX, Chen SP, Li YD, Wang J, Zhang B, Lin Y, Guan JH, Cai YF, Liang Z, Zheng F (2015) The Effect of the modified eighth section of eight-section brocade on osteoporosis in postmenopausal women: a prospective randomized trial. Medicine 94:e991

34. Yamazaki S, Ichimura S, Iwamoto J, Takeda T, Toyama Y (2004) Effect of walking exercise on bone metabolism in postmenopausal women with osteopenia/osteoporosis. J Bone Miner Metab 22:500-508

35. Greenway KG, Walkley JW, Rich PA (2015) Impact exercise and bone density in premenopausal women with below average bone density for age. Eur J Appl Physiol 115:2457-2469

36. Niu K, Ahola R, Guo H et al (2010) Effect of office-based brief high-impact exercise on bone mineral density in healthy premenopausal women: the Sendai Bone Health Concept Study. J Bone Miner Metab 28:568-577

37. Iwamoto J, Takeda T, Ichimura S (2001) Effect of exercise training and detraining on bone mineral density in postmenopausal women with osteoporosis. J Orthop Sci 6:128-132

38. Ebrahim S, Thompson PW, Baskaran V, Evans K (1997) Randomized placebo-controlled trial of brisk walking in the prevention of postmenopausal osteoporosis. Age Ageing 26:253-260

39. Brooke-Wavell K, Jones PR, Hardman AE (1997) Brisk walking reduces calcaneal bone loss in post-menopausal women. Clin Sci 92:75-80 
40. Bouxsein ML, Eastell R, Lui LY, Wu LA, de Papp AE, Grauer A, Marin F, Cauley JA, Bauer DC, Black DM (2019) Change in bone density and reduction in fracture risk: a meta-regression of published trials. J Bone Miner Res 34:632-642

41. Eastell R, Vittinghoff E, Lui LY, McCulloch CE, Pavo I, Chines A, Khosla S, Cauley JA, Mitlak B, Bauer DC (2021) Validation of the surrogate threshold effect for change in bone mineral density as a surrogate endpoint for fracture outcomes: the FNIH-ASBMR SABRE Project. Journal of Bone and Mineral Research

42. Black DM, Bauer DC, Vittinghoff E, Lui L-Y, Grauer A, Marin F, Khosla S, de Papp A, Mitlak B, Cauley JA (2020) Treatmentrelated changes in bone mineral density as a surrogate biomarker for fracture risk reduction: meta-regression analyses of individual patient data from multiple randomised controlled trials. Lancet Diabetes Endocrinol 8:672-682

43. Rutherford OM (1999) Is there a role for exercise in the prevention of osteoporotic fractures? Br J Sports Med 33:378-386

44. Kemmler W, Bebenek M, Kohl M, Von Stengel S (2015) Exercise and fractures in postmenopausal women. Final results of the controlled Erlangen Fitness and Osteoporosis Prevention Study (EFOPS). Osteoporos Int 26:2491-2499

45. Daly RM, Dalla Via J, Duckham RL, Fraser SF, Helge EW (2019) Exercise for the prevention of osteoporosis in postmenopausal women: an evidence-based guide to the optimal prescription. Braz J Phys Ther 23:170-180

46. Vainionpää A, Korpelainen R, Sievänen H, Vihriälä E, Leppäluoto J, Jämsä T (2007) Effect of impact exercise and its intensity on bone geometry at weight-bearing tibia and femur. Bone 40:604-611

47. Sánchez-Trigo H, Sanchez-Oliver AJ, Abt G, Sañudo B (2020) Validation of a wearable accelerometer-based activity monitor for use in future osteoporosis prevention programs. Sustainability $12: 2187$

48. Giangregorio L, Papaioannou A, Macintyre N, Ashe M, Heinonen A, Shipp K, Wark J, McGill S, Keller H, Jain R (2014) Too fit to fracture: exercise recommendations for individuals with osteoporosis or osteoporotic vertebral fracture. Osteoporos Int 25:821-835

49. Watson SL, Weeks BK, Weis LJ, Harding AT, Horan SA, Beck BR (2018) High-intensity resistance and impact training improves bone mineral density and physical function in postmenopausal women with osteopenia and osteoporosis: the LIFTMOR randomized controlled trial. J Bone Miner Res 33:211-220

50. Kistler-Fischbacher M, Weeks BK, Beck BR (2021) The effect of exercise intensity on bone in postmenopausal women (part 2): a meta-analysis. Bone 143:115697

51. Tanaka H, Tarumi T, Rittweger J (2011) Aging and physiological lessons from master athletes. Compr Physiol 10:261-296

52. Suominen T, Alén M, Heinonen A, Degens H, Rittweger J, Törmäkangas T, Suominen H, Korhonen M (2020) Regular strength and sprint training counteracts bone aging: a 10-year follow-up in male masters athletes. J Bone Min Res 35:155-156

53. Ireland A, Mittag U, Degens H, Felsenberg D, Ferretti JL, Heinonen A, Koltai E, Korhonen MT, McPhee JS, Mekjavic I (2020) Greater maintenance of bone mineral content in male than female athletes and in sprinting and jumping than endurance athletes: a longitudinal study of bone strength in elite masters athletes. Arch Osteoporos 15:1-10
54. Wooten SV, Mittag U, Alvero Cruz JR, Stray-Gundersen S, Hoffmann F, Michély S, Möstl S, Sies W, Mulder E, Rauschendorfer P (2021) Life satisfaction, positive affect, and sleep impairment in masters athletes: modulation by age, sex, and exercise type. Front Physiol 12:109

55. Moreira LDF, Oliveira MLd, Lirani-Galvão AP, Marin-Mio RV, Santos RNd, Lazaretti-Castro M (2014) Physical exercise and osteoporosis: effects of different types of exercises on bone and physical function of postmenopausal women. Arquivos Brasileiros de Endocrinologia \& Metabologia 58:514-522

56. Martyn-St James M, Carroll S (2010) Effects of different impact exercise modalities on bone mineral density in premenopausal women: a meta-analysis. J Bone Miner Metab 28:251-267

57. Bonaiuti D, Shea B, Iovine R, Negrini S, Welch V, Kemper HH, Wells GA, Tugwell P, Cranney A (2002) Exercise for preventing and treating osteoporosis in postmenopausal women. Cochrane Database of Systematic Reviews

58. Kasper MJ, Peterson MG, Allegrante JP (2001) The need for comprehensive educational osteoporosis prevention programs for young women: results from a second osteoporosis prevention survey. Arthritis Care Res: Official J Am College Rheumatol 45:28-34

59. Bailey DA, Faulkner RA, McKay HA (1996) Growth, physical activity, and bone mineral acquisition. Exerc Sport Sci Rev 24:233-266

60. Kemmler W, Shojaa M, Kohl M, von Stengel S (2020) Effects of different types of exercise on bone mineral density in postmenopausal women: a systematic review and meta-analysis. Calcified Tissue International 1-31

61. Macías-Hernández SI, Loya-García F, Zepeda-Mora R, NavaBringas TI, Morones-Alba JD (2020) Disease-related factors associated with exercise adherence in postmenopausal women with osteoporosis. J Frailty, Sarcopenia Falls 5:72

62. Simmonds BA, Hannam K, Fox KR, Tobias JH (2016) An exploration of barriers and facilitators to older adults' participation in higher impact physical activity and bone health: a qualitative study. Osteoporos Int 27:979-987

63. Bachmann C, Oesch P, Bachmann S (2018) Recommendations for improving adherence to home-based exercise: a systematic review. Physikalische Medizin, Rehabilitationsmedizin, Kurortmedizin 28:20-31

64. Valenzuela T, Okubo Y, Woodbury A, Lord SR, Delbaere K (2018) Adherence to technology-based exercise programs in older adults: a systematic review. Journal of Geriatric Physical Therapy 41:49-61

65. Tate DF, Lyons EJ, Valle CG (2015) High-tech tools for exercise motivation: use and role of technologies such as the internet, mobile applications, social media, and video games. Diabetes Spectrum 28:45-54

66. Esteves SC, Majzoub A, Agarwal A (2017) The problem of mixing "apples and oranges" in meta-analytic studies. Transl Androl Urol 6:S412-S413

Publisher's note Springer Nature remains neutral with regard to jurisdictional claims in published maps and institutional affiliations. 